\title{
Un modèle lagrangien de qualité. Application à l'Arc à l'aval d'Aix-en-Provence
}

\author{
J. M. Mouchel ('), M. Z. Zhang ( $\left.{ }^{1}\right)$, X. Bourrain ( $\left.{ }^{2}\right)$ et B. André ( $\left.{ }^{3}\right)$
}

\section{Introduction}

En principe, la modélisation mathématique est une technique utilisée pour la gestion de la qualité des eaux, un des critères de choix d'un aménagement étant lamélioration attendue de la qualité des eaux du milieu récepteur calculée à partir d'un modèle mathématique théoriquement capable de faire des prédictions. En pratique, en France, des modèles ont effectivement été utilisés dans les programmes d'objectifs de qualité en rivière et sont encore utilisés pour des études ponctuelles, mais ne servent-ils pas le plus souvent d'outil pédagogique, voire d'alibi, pour des aménagements dont l'installation est en fait dictée par l'expérience des gestionnaires et limitée par des contraintes techniques, financières ou politiques. Or, face à ces usages limités, on assiste au développement de très nombreux modèles. Individuellement peu différents de leurs voisins, mais qui dans l'ensemble fournissent un très large spectre d'outils potentiels.

La contradiction n'est qu'apparente, car il faut distinguer d'une part les modèles de recherche, jamais achevés, destinés à comprendre de plus en plus finement le fonctionnement des écosystèmes, d'autre part les modèles de gestion, destinés à l'analyse de l'efficacité d'aménagements et qui doivent pouvoir fonctionner sur la base de données souvent limitées, sans possibilité de retour sur le terrain pour vérifications et mesures complémentaires.

L'étude préalable d'un aménagement devrait s'effectuer en deux temps, en suivant la démarche standardisée des études d'impact: d'abord une analyse du système récepteur, puis l'investigation de l'effet attendu de différents aménagements. Les deux parties de la démarche ne sont pas forcément basées sur les mêmes outils : l'outil d'analyse doit être adaptable aux situations les plus diverses (débits et concentrations pouvant fortement fluctuer), sans requérir les services d'un spécialiste de l'analyse numérique, il permet de juger de l'importance de différents processus dans le milieu récepteur; au contraire, l'outil de prédiction fonctionnera en règle générale sur la base d'hypothèses de travail, moins tourmentées que les fluctuations naturelles de débit et de concentration. Par contre, l'outil de prédiction doit être relié à une base de données qui intègre l'expérience acquise sur d'autres sites, et permet des extrapolations acceptables.

L'objectif de cet article est de présenter la technique lagrangienne, de mettre en évidence ses avantages et ses inconvénients, et de montrer quel peut être son intérêt, par rapport à d'autres méthodes, pour la gestion de la qualité des eaux. Nous pensons qu'elle peut être la base d'un bon outil d'analyse dans les cas mono-dimensionnels.

\section{Intérêt de la technique lagrangienne}

Les instabilités et la dispersion numérique sont les plaies de la résolution numérique de l'équation d'advection. En effet, ces difficultés conduisent à des contraintes numériques sur les pas d'espaces et les pas de temps, qui génèrent des temps de calcul et des besoins en mémoire élevés, et doivent être étudiées dans la préparation des simulations. Ces problèmes ne devraient pas être ceux du gestionnaire qui utilise un modèle.

Grossièrement, on peut séparer les techniques de résolution de l'équation d'advection en deux catégories (Simon 1989): les schémas aux différences finies et les

(') CERGRENE, Ecole nationale des Ponts et Chaussées.

( ${ }^{2}$ ) LEMAT, Université de Dijon (adresse actuelle : Agence de bassin Seine-Normandie, délégation de ChampagneArdenne).

( $\left.{ }^{3}\right)$ Agence de bassin Rhône-Méditerranée-Corse.

\section{A lagrangian model for water quality modelling}

This paper presents the lagrangian technique, its advantages and limitations. Its principle is to follow the information provided by the upstream boundary conditions are the lateral inputs downstream the river. It is a very rapid technique with very little numerical problems such as constraints on the time and space steps. It may be the basis of reliable water management tools, including automated calibration, and systematic sensitivity analysis. 
techniques aux caractéristiques. La plupart des schémas aux différences finies souffrent des mêmes défauts, plus ou moins accentués selon la technique spécifique mise en œuvre. Ils imposent, pour des raisons de stabilité, un nombre de Courant $(U . \Delta t / \Delta x)$ compris dans des domaines restreints, autour de l'unité en général. De plus, aux forts nombres de Peclet $(U . \Delta x / D)$ (advection prépondérante sur la dispersion), les schémas qui évitent une trop forte dispersion numérique provoquent des instabilités : pour éviter que les fronts de concentration ne s'écrasent, ces schémas génèrent des concentrations trop fortes ou trop faibles immédiatement à l'amont ou à l'aval du front. Le dosage entre les deux contraintes (stabilité et dispersion numérique) est très délicat et fait que ces schémas ne sont pas facilement utilisables, ni adaptables à des situations complexes. Les schémas aux caractéristiques par contre ne souffrent plus de problèmes d'instabilité mais la dispersion numérique (lissage des concentrations) causée par un trop grand nombre d'interpolations reste un problème lorsque les pas de temps sont trop petits.

Face à ces difficultés, les énormes avantages des schémas lagrangiens sont qu'ils ne génèrent aucune dispersion numérique, puisqu'aucune interpolation des concentrations n'est faite, qu'ils permettent d'ajuster le nombre de points de calcul à l'information disponible sur le nuage polluant, limitant donc le temps de calcul au strict nécessaire, et qu'ils n'imposent aucune contrainte sur les pas de temps et d'espace.

Les contraintes des schémas classiques sur les pas de temps et d'espace sont particulièrement gênantes dans le cas d'apports brutaux de pollution. En effet, une prise en compte précise de l'apport impose des pas de temps petit, et le suivi du nuage polluant au cours de son transit dans la rivière impose des pas d'espace également petits. Or, si les mailles et le pas de temps sont figés, on est obligé d'utiliser un pas de temps et un maillage fins pour toute la simulation et sur toute la longueur de la rivière. $\mathrm{Au}$ contraire, pour traiter ce problème, la technique lagrangienne définira autant de points qu'il est nécessaire pour générer le nuage initial, puis on suivra ces points au cours de leur transit le milieu.

En dehors du cas des rejets brutaux, la souplesse de la technique lagrangienne peut également être appréciable dans la partie "analyse " d'une étude d'aménagement, car les données de terrain peuvent amener à des contraintes sévères sur les pas d'espace et de temps, s'il n'est pas possible d'utiliser des données moyennées, en se ramenant à un état quasi-stationnaire, pour analyser les processus ayant cours dans le milieu. En effet, il suffit alors de résoudre une équation aux dérivées droites, la dispersion pouvant être négligée (Fisher 1979).

L'utilisation de données moyennées est licite si les cinétiques employées dans le modèle sont linéaires ou quasi-linéaires, ce qui est assez souvent le cas en rivière : la $\mathrm{DBO}$ et le déficit en Oxygène entre autres sont exactement linéaires par rapport aux apports, et lorsque des cinétiques sont non linéaires, en général de type Monod, la non-linéarité n'apparaît que dans des cas d'extrême appauvrissement du milieu, assez rares en rivière. Paradoxalement, si toutes les cinétiques sont linéaires, il n'est même plus nécessaire de faire tourner le moindre modèle puisque les concentrations après aména- gement pourront être obtenues par de simples règles de trois.

Une technique plus élaborée ne devra donc être employée que dans les cas où des fluctuations de débit rendent difficile le suivi des masses d'eau, où le moyennage des mesures ne provoque pas un lissage suffisant des concentrations, où des non-linéarités sont attendues. Dans ces cas là, pour des raisons de performance numérique et de souplesse d'utilisation, on aura intérêt à utiliser la technique lagrangienne.

\section{Exposé de la méthode}

\section{a) Transformation de l'équation d'advection dispersion dans un repère lagrangien}

Le principe est similaire à celui qu'a utilisé O'Kane (1974) pour définir un système de coordonnées mobiles en estuaire à marée, on utilise la transformation $\phi(x, t)$ qui associe à un point $x$ à l'instant $t$ la position de la particule d'eau se trouvant en ce point à l'instant $t$, en un instant de référence : $t_{0}$. Une propriété fondamentale de $\phi$ est : $u(x, t) \partial \phi / \partial x+\partial \phi / \partial t=0$; car en suivant un point dans son mouvement, la position initiale ne change pas. $\partial \phi / \partial x$ caractérise la distorsion du référentiel lagrangien. L'équation d'advection diffusion

$$
\frac{\partial c}{\partial t}+u(x, t) \frac{\partial c}{\partial x}=(1 / A) \frac{\partial}{\partial x}\left(A D\left(\frac{\partial c}{\partial x}\right)\right)
$$

devient après changement de référentiel :

$$
\frac{\partial c}{\partial t}=(1 / A)\left(\frac{\partial \phi}{\partial x}\right)^{2} \cdot \frac{\partial}{\partial X}\left(A D \frac{\partial c}{\partial X}\right)+D \cdot \frac{\partial c}{\partial X} \cdot \frac{\partial^{2} \phi}{(\partial x)^{2}}
$$

Deux observations doivent être faites à l'issue de cette transformation.

a) Le coefficient de dispersion est modifié par rapport au coefficient en coordonnées euleriennes, en négligeant les variations longitudinales de $A$ (la section mouillée) et $D$ (le coefficient de dispersion), on peut écrire que :

$$
D_{L}=(\partial \phi / \partial x)^{2} \cdot D_{E}
$$

où $D_{L}$ est le coefficient de dispersion en coordonnées lagrangiennes et $D_{E}$ le coefficient en coordonnées euleriennes. $\partial \phi / \partial x$ est en fait égal à $A(x, t) / A\left(\phi(x, t), t_{0}\right)$ car en coordonnées lagrangiennes le volume d'eau situé entre deux points ne change pas avec le temps, donc le rapport des distances entre deux points très proches est l'inverse du rapport des sections mouillées.

b) La deuxième observation est plus gênante. Un terme advectif (en $\partial c / \partial X$ ) est apparu, alors que la transformation était destinée à les faire disparaître. O'Kane (1974) indique que ce terme peut être négligé en estuaire, en raison des faibles évolutions longitudinales des sections mouillées, néanmoins, dans le cas général, en rivière, on ne pourra pas le négliger. La transformation lagrangienne de l'équation d'advection-dispersion n'est donc pas complètement intéressante. 


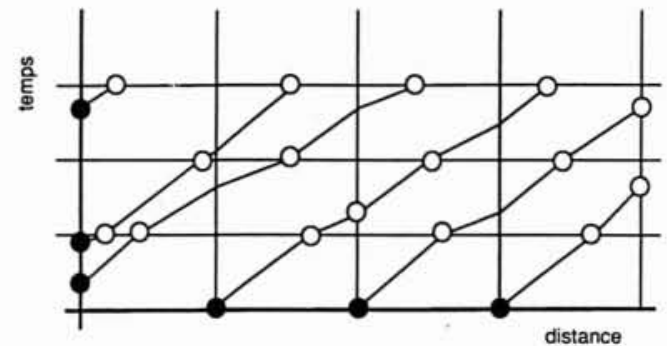

1. Principe de la technique lagrangienne.

Les points figurent les informations fournies au modèle (état initial ou apport amont à gauche). Les cercles figurent les points où le modèle estime une concentration. Les trajectoires sont calculées à partir des vitesses qui doivent être connues sur la grille.

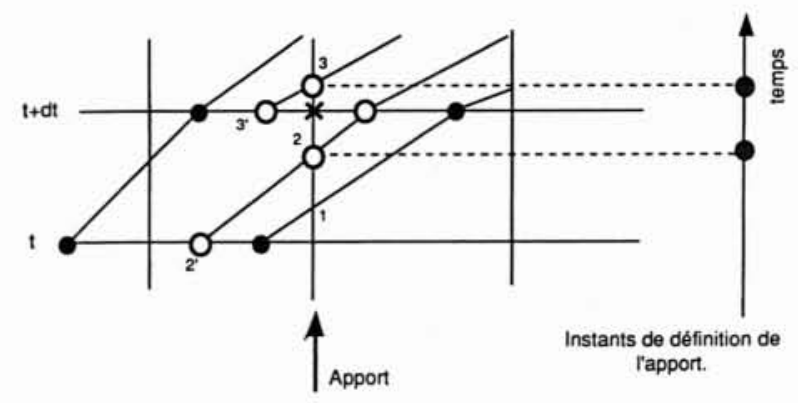

2. Gestion des apports.

Les points noirs figurent les trajectoires préexistantes avant la prise en compte de l'apport. Les points grisés figurent les instants pour lesquels la concentration est connue dans l'apport. Au point 1, la concentration sur la trajectoire est modifiée en fonction de lapport. Aux points 2 et 3 de nouvelles trajectoires sont générées pour tenir compte de l'information supplémentaire due à l'apport. La croix figure un point temporaire destiné à éviter une interférence entre les calculs des concentrations pour les trajectoires (2) et (3).

\section{b) Le schéma pseudo-lagrangien}

Notre schéma n'est que pseudo-lagrangien, il s'agit en fait d'un schéma similaire aux techniques aux caractéristiques, où l'on descend les courbes caractéristiques au lieu de les remonter. Donc, plutôt que d'une résolution en coordonnées lagrangiennes de l'équation d'advectiondispersion, il s'agit d'un schéma fractionnaire où, à chaque pas de temps, l'étape d'advection est résolue par une technique lagrangienne qui permet d'éviter les interpolations, les autres étapes étant résolues avec des techniques standard.

Le principe est schématisé sur la figure 1 . Il consiste à suivre dans leur mouvement un jeu de points mobiles dont la concentration est connue, soit à partir des concentrations initiales, soit après un apport, de leur appliquer les cinétiques chimiques adéquates et de procéder à un calcul de dispersion à chaque pas de temps. Par rapport aux schémas aux caractéristiques, les interpolations sont presque totalement supprimées. Elles sont réservées aux sorties, si l'utilisateur demande les concentrations en un point et un instant précis (on aura alors à faire une interpolation pour calculer les concentrations à partir des points voisins), ou aux apports et aux jonctions, lorsqu'un nouveau point doit être créé (sa concentration est calculée une fois par interpolation avant que le point rejoigne le train des points mobiles).

En pratique, il existe deux techniques lagrangiennes. La première, développée par Jobson et Schoellhamer (JOBSON et SCHOELlHAMER 1987, JOBSON 1987, SCHOELLHAMER 1988) et MCBRIDE et RUTHERFORD (1985), transporte des boîtes de concentration homogène en calculant le déplacement des frontières de chaque boîte. L'avantage de cette technique est qu'elle assure la conservativité des quantités transportées, par contre, les cinétiques chimiques étant calculées en moyenne sur une boîte, elle ne permet pas de rendre compte facilement de phénomènes locaux comme une réaération sur un seuil. La deuxième technique est celle que nous employons: des points sont transportés et non plus des boîtes. Elle permet une description plus fine des cinétiques en relation avec le milieu environnant, mais on n'a aucune garantie de conservativité des quantités transportées.

Avec ce schéma pseudo-lagrangien, les pas d'espace et les pas de temps ne servent qu'à calculer les trajectoires, et à résoudre l'étape de dispersion, aucune contrainte ne provient donc du terme d'advection. Dans le cas extrême où les vitesses sont uniformes en temps et en espace, et où la dispersion est négligeable, on pourrait travailler avec un seul pas d'espace et un seul pas de temps qui couvrent tout le domaine de la simulation.

\section{Particularités. Principales difficultés}

\section{a) Gestion des apports}

Un apport est constitué d'une série de $n$-uplets (temps, débit, concentration). Pour chaque point mobile qui traverse une section d'apport, on calcule une nouvelle concentration par une formule de mélange : au point 1 (fig. 2) la concentration sur la trajectoire est modifiée selon la formule $c=\left(q \cdot c+q_{A} \cdot c_{A}\right) /\left(q+q_{A}\right)$. En outre, pour chaque instant de définition de l'apport (point 2), appartenant à la série des $n$-uplets, on va générer un point mobile supplémentaire, une remontée de caractéristique permettant de calculer par interpolation la concentration de ce nouveau point mobile au début du pas de temps. Ainsi, conformément à la philosophie de la technique lagrangienne, on ne transporte tous les points et seulement les points pour lesquels on dispose d'une information.

Néanmoins, on rencontre une difficulté au point 3, car la concentration sur la caractéristique au début du pas de temps suivant (point $3^{\prime}$ ) ne doit pas être calculée par une interpolation basée sur un point (aval) déjà sous l'influence de l'apport. Donc on utilise des points complémentaires ajoutés au niveau de chaque apport, qui ne seront pas transportés ultérieurement, mais qui garantissent l'existence au pied de chaque apport et au début du pas de temps d'une valeur de concentration qui ne soit pas déjà sous l'influence de l'apport. 


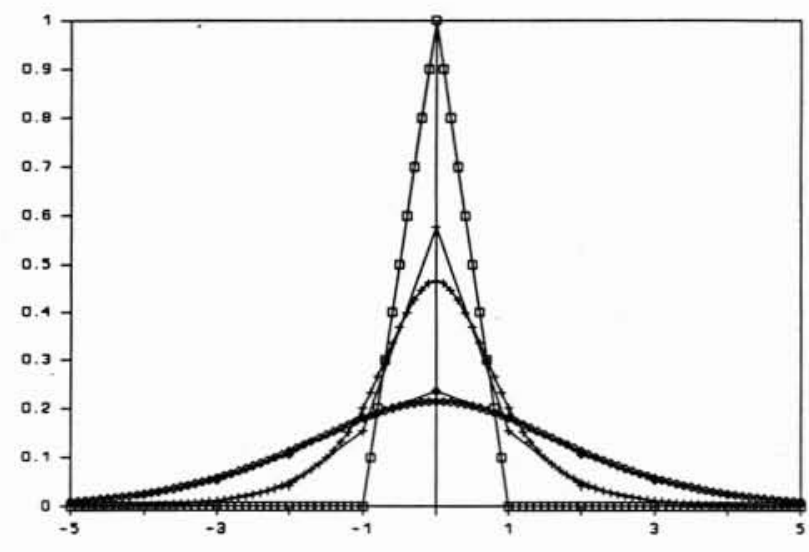

3. Comparaison de deux grilles de calcul pour le même pic. Les pas d'espace des deux grilles diffèrent d'un facteur 10. Il apparaît que la grille la plus lâche (pic défini en un seul point) ne provoque pas d'erreur de calculs graves. Carrés : initial ; croix : 1 itération; losanges : 4 itérations.

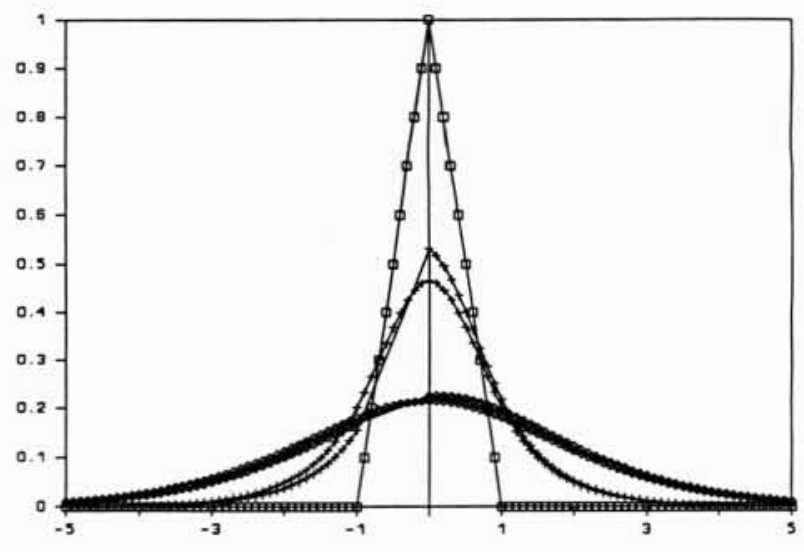

4. Influence de l'irrégularité de la grille.

Pour une des deux simulations, l'aile gauche du pic n'est définie que par un point. Carrés : initial ; croix : 1 itération ; losanges : 4 itérations. ici encore, les erreurs sont très faibles. b) Gestion des convergences et des divergences

Le même protocole peut être appliqué : pour chaque point mobile d'une branche quelconque, on calcule par interpolation les concentrations des autres branches au lieu de confluence, à l'instant où le premier point arrive. Comme pour la gestion des apports, on doit prévoir des points complémentaires, à la fin de chaque tronçon, pour que les interpolations aux convergences conduisent à des résultats corrects.

Dans le cas de la technique lagrangienne avec transport de boîtes et non plus de points, ces problèmes sont moins délicats à résoudre. En effet, à chaque jonction, on fabrique de nouvelles boîtes basées sur les instants d'arrivée des frontières des boîtes provenant des tronçons amont, les concentrations dans ces boîtes sont obtenues par simple mélange.

\section{c) Dispersion}

Puisque le schéma considéré n'est pas une véritable résolution de l'équation d'advection dans un référentiel lagrangien, mais une résolution de l'étape d'advection par une technique lagrangienne, le coefficient de dispersion ne doit pas être modifié par un terme de distorsion, on utilise donc le coefficient de dispersion eulerien.

Par contre deux types de problèmes sont possibles.

a) Nous pouvons avoir à faire à des concentrations qui varient brutalement d'un point à un autre, car d'importantes fluctuations des apports pourront n'être décrites que par un seul point, à savoir la mesure.

b) La grille de résolution de l'équation de dispersion, définie par la position des points mobiles en fin de pas de temps, peut être très hétérogène; et nous savons que l'erreur du schéma standard en trois points d'approximation du terme de dispersion est de l'ordre de $\left(\Delta x_{l+1}-\Delta x_{l}\right)$.

L'analyse de Von Neumann indique que le schéma en trois points d'estimation de la dérivée seconde sera stable si le coefficient d'implicitation $\left(\Theta_{D}\right)$ est supérieur à 0,5 , cependant, dans le cas de dispersion forte (soit $\left.Z=D . \Delta t /(\Delta x)^{2}>1\right)$, on observe des oscillations, qui s'amortissent puisque le schéma est stable, mais qui peuvent provoquer des concentrations négatives. Pour supprimer ces aberrations, on peut utiliser une technique de résolution totalement implicite $\left(\Theta_{D}=1\right)$ qui fournit des résultats proches de la résolution semi-implicite (théoriquement plus précise) lorsque $Z \ll 1$ au bout de une itération, et presque impossibles à distinguer au bout de quelques itérations. De plus, la comparaison avec une solution implicite obtenue avec des pas d'espace plus petits est aussi presque égale (fig. 3), donc, le fait de ne pas avoir de points intermédiaires pour définir des pics de concentration n'est pas un handicap.

Comme les pas d'espaces et les pas de temps peuvent être tout à fait divers, et que l'objectif de la technique lagrangienne est de pouvoir les choisir sans contrainte numérique, nous utilisons une résolution implicite, qui ne génère que de faibles erreurs qui disparaissent après quelques pas de temps.

Il n'est pas possible d'analyser pour tous les cas possibles d'impact de l'irrégularité des pas d'espaces, d'autant plus qu'ils sont modifiés à chaque pas de temps ; aucune solution de référence ne peut donc être établie. Nous avons donc choisi de tester un cas d'irrégularité forte ou deux pas d'espace consécutifs varient d'un facteur 10, les résultats sont portés sur la figure 4 . L'irrégularité des pas d'espace provoque une distorsion de la courbe qui va s'amenuisant avec le nombre des itérations.

En résumé, le faible nombre de points de définition des pics et l'existence de pas irréguliers provoquent des erreurs dans l'amplitude des pics après la première itération suivant l'apparition du pic, ces erreurs sont très rapidement atténuées au bout de quelques itérations.

\section{d) Echange avec des zones mortes}

Si la concentration dans des zones mortes ou des échanges avec le fond doivent être simulés, la technique lagrangienne risque de poser des problèmes. En effet, les points mobiles pouvant être très éloignés les uns des autres en fonction de la répartition des informations dans les apports, on peut, à une abscisse donnée, observer de longues périodes sans informations sur les concentrations dans la masse d'eau, et être dans l'incapacité d'estimer correctement les flux vers le fond ou les zones mortes.

$\mathrm{Si}$ les longues périodes sans données proviennent en 
fait de l'absence de mesures sur les apports, l'insuffisance ne provient pas de la technique lagrangienne, mais du protocole d'échantillonnage. Au contraire, dans le cas d'apports presque constants, l'utilisateur peut volontairement limiter le nombre de points de définition des apports, l'insuffisance provient alors effectivement de la technique lagrangienne. Nous mettons ici en évidence le revers des avantages de cette technique: en voulant suivre les masses d'eau le plus précisément possible, on est dans l'incapacité de fournir une information précise en une abscisse donnée.

Une solution au problème est de générer assez de points mobiles (à l'amont par exemple) pour que les différences de concentration entre deux points successifs restent assez faibles. On perd donc un des avantages de la technique lagrangienne, par rapport aux techniques aux caractéristiques, qui était de limiter le nombre de points de calcul pour s'ajuster au plus prêt aux informations disponibles, par contre, on conserve l'avantage lié à l'absence de dispersion numérique. Néanmoins, si les mailles de définition des zones mortes sont trop grandes par rapport aux distances séparant les points mobiles, elles génèreront une dispersion numérique supplémentaire d'autant plus forte que les échanges sont de grande intensité. En outre, ces échanges ne peuvent être traités qu'explicitement, ce qui imposent qu'ils soient suffisamment faibles au cours d'un pas de temps pour ne pas provoquer d'instabilités.

Il est également possible de traiter le cas de tubes de courant de vitesses différentes par une technique lagrangienne, les problèmes d'échanges entre zones de vitesses différentes sont alors tout à fait similaires aux problèmes d'échanges avec zones mortes; il est nécessaire de garantir une répartition suffisamment dense des points mobiles le long de chaque tube, par rapport aux variations de concentration entre deux points successifs, et l'intensité des échanges doit être limitée.

Dans le cas de la technique lagrangienne avec transport de boîtes, les problèmes d'échange avec le fond sont moins complexes puisqu'on sait définir une concentration en tout point à tout instant (tout point appartenant à une boîte), néanmoins, les problèmes de dispersion numérique et de stabilité restent entiers.

\section{e) Suppression de points}

Pour des raisons de vitesse de calcul et d'encombrement de la mémoire, il peut être utile de supprimer des points mobiles lorsque l'information qu'ils véhiculent a perdu de son intérêt, notamment en raison de la dispersion. La décision de suppression d'un point doit être fondée sur deux critères: d'une part le volume des deux mailles mobiles situées autour de ce point (et non pas leur taille car elle peut changer), d'autre part la linéarité de toutes les concentrations autour de ce point.

\section{Conclusions sur la technique lagrangienne}

La technique lagrangienne est incontestablement la plus précise pour résoudre l'équation d'advection dispersion à une dimension, elle ne génère aucune dispersion numérique ni instabilités. En outre, elle est très économi- que car on ne transporte que les points pour lesquels on dispose d'une information à véhiculer, information apportée par un point de rejet ou des mesures à l'amont. Enfin, elle n'impose aucune contrainte de pas d'espace ou de pas de temps.

Dans la pratique, les seules contraintes de pas d'espace et de pas de temps proviennent de l'hydraulique (les vitesses ne doivent pas trop varier dans une maille pour que le calcul des trajectoires soit précis), de l'étape de dispersion (mais cette contrainte n'est pas trop gênante) et des cinétiques chimiques car, malgré toute la puissance des méthodes de type Runge-Kutta, les pas de temps doivent rester adaptés aux cinétiques et aux forçages. Elles sont beaucoup moins sévères que les contraintes sur le nombre de Courant des schémas aux différences finies.

La technique lagrangienne permet de transporter des points ou des boîtes, ces deux variantes sont numériquement très proches. Le transport de points est légèrement plus délicat, essentiellement dans la gestion des jonctions et des apports, par contre, il permet de mieux prendre en compte des cinétiques chimiques évoluant en fonction de la position, ou des non-linéarités.

Par contre, son très gros défaut est d'être absolument inadaptée aux problèmes à plusieurs dimensions, elle peut seulement être utilisée dans le cas de plusieurs tubes de courant parallèles, à la condition que les échanges entre tubes ou avec le fond soient de faible intensité car ils devront être traités explicitement.

Dans la pratique de la gestion des eaux, elle devra être utilisée dans les cas où l'hydraulique ou les apports sont fortement fluctuants ou réduits, voire réduits à un seul rejet brutal. Dans ces cas, elle est particulièrement adaptée aux campagnes de mesure "en suivant les masses d'eau ". Elle constitue alors un outil de choix pour le gestionnaire en raison de sa souplesse d'utilisation et des faibles contraintes numériques qu'elle impose.

\section{Un exemple d'application : L'Arc à l'aval d'Aix- en-Provence}

L'Arc est un fleuve côtier qui débouche dans l'étang de Berre. Son cours couvre $87 \mathrm{~km}$, dont environ 40 à l'aval d'Aix-en-Provence, où sa pente est de 0,003 , et sa largeur de 12 à $15 \mathrm{~m}$. A l'étiage, son débit est d'environ $1 \mathrm{~m}^{3} / \mathrm{s}$. La station d'épuration de la Pioline, qui traite les eaux usées de la ville d'Aix a un débit moyen journalier supérieur à $300 \mathrm{l} / \mathrm{s}$, elle contribue donc considérablement à la fois à l'hydrologie et à la qualité des eaux de l'Arc (Bourrain 1988).

Plus en aval, quelques apports de moindre importance ont également été pris en compte (petits affluents et stations d'épuration de moindre importance (environ 10000 eq. hab. au total). Une importante prise d'eau existe au niveau de Moulin du Pont (environ $15 \mathrm{~km}$ à l'aval d'Aix), destinée à l'irrigation. Les rares surplus sont rejetés à l'Arc au niveau de La Fare. C'est à La Fare également que se situe la prise d'eau d'alimentation des usines de Berre.

Tout au long du cours, on observe une invasion de macrophytes (Potamogeton Pectinatus) et d'algues fila- 
menteuses de type Cladophore qui influencent la qualité de l'eau, en consommant de l'Azote et du Phosphore et en provoquant de fortes variations journalières de l'Oxygène dissous et du $\mathrm{pH}$.

Les fluctuations journalières, en débit et en qualité, du rejet de la station provoquent des variations de concentration très importantes dans l'Arc (un facteur 3), auxquelles viennent s'additionner les cycles journaliers de la photosynthèse. On est donc dans un cas de forte instationnarité, et un modèle capable de simuler le transport avec précision devait être utilisé.

Dans une première étape, il était nécessaire de caler la partie hydraulique et transport du modèle. Deux traçages ont été effectués (moins chers et plus efficaces qu'un relevé précis des sections de la rivière) dans des conditions de débit légèrement différentes. La forme des sections étant mal connue et de toutes façons éminemment variable, nous avons utilisé une géométrie simple et calé le paramètre de frottement pour obtenir des vitesses d'eau adéquates.

Ensuite, nous avons calibré les cinétiques ( $3 \mathrm{~min}$ de temps calcul pour une simulation de 5 jours sur un COMPAQ 386/20), à partir des données d'une campagne réalisée en septembre 1987. Elle a permis de vérifier la forte variabilité des concentrations, qui s'observe aussi en moyenne d'un jour à l'autre (week-ends, variation des débits de l'Arc qui provoque une modification des dilutions des rejets...). Les cinétiques utilisées sont simples: ordre 1 pour la dégradation de la DBO carbonée et les deux étapes de nitrification, relargages constants d'ammonium et consommation benthique d'oxygène (estimés à partir de mesures directes de consommation d'oxygène par des petites carottes de sédiment), la respiration et consommation d'azote par les végétaux sont des constantes surfaciques, et la photosynthèse suit un morceau de sinusoïde durant la journée. La stoechiométrie de la respiration, de la photosynthèse et de la consommation d'azote par les végétaux (et l'épiphyton associé) a également été mesurée au laboratoire (Bourrain 1988). Par contre, la biomasse totale des végétaux n'a pas pu être estimée de façon satisfaisante dans l'Arc en raison de difficultés d'accès à la rivière ; elle a donc été calée.

Puis, les coefficients ont été vérifiés grâce aux données d'une autre campagne de mesure (juillet 1988), offrant des conditions très différentes (flux diminués de plus du tiers), puisque la population avait beaucoup diminué à Aix. Quelques observations ont montré un accroissement de la quantité de végétaux, estimé à $25 \%$.

Sans entrer dans les détails, la vérification a donné des résultats acceptables en moyenne. L'utilisation d'un modèle de transport correct a permis de mieux situer, malgré l'incertitude de mesure des débits, la position des instants de mesure aux différents points aval en fonction de la fluctuation des rejets de la station. Elle a également permis de mettre en évidence des évolutions de concentrations qui n'étaient pas dues aux processus de transport et dispersion, notammęnt un lissage des concentrations de nitrates, par rapport aux concentrations d'ammonium initiales. Ce lissage indique un stockage des pics suivi d'un relargage plus régulier, sans qu'il soit possible à l'heure actuelle de préciser son origine.

Le modèle, outil d'analyse, a permis de mieux comprendre et évaluer certains aspects du fonctionnement biogéochimique de l'Arc. Il a été un partenaire de l'étude de terrain, en permettant d'aider à la définition des campagnes de mesure et de les interpréter plus finement. Finalement, après la phase d'analyse du système, nous avions une estimation correcte des principales cinétiques de l'Arc et de leurs paramètres.

Par la suite, il a également été utilisé comme outil de prédiction: différents scénarii d'aménagement ont pu être testés (nitrification ou dénitrification à la station d'Aix-en-Provence...) et d'autres le seront dans un proche avenir. Néanmoins, la finesse fournie par la technique lagrangienne n'est pas toujours nécessaire, et d'autres outils, plus rapides, pourraient être utilisés au niveau de la phase de prédiction. L'estimation de certains paramètres susceptibles de varier à la suite d'aménagements est une partie délicate du problème de la prédiction, parfois assez sensible au niveau des concentrations prédites. Nous pensons qu'une utilisation plus exhaustive de modèles lors des études, associées à une base de données, permettrait dans l'avenir de faciliter ces estimations. En effet le modèle oblige à formaliser l'ensemble des mesures de terrain ou de laboratoire, une fois cette formalisation faite, chaque étude de cas pourrait probablement assez facilement être intégrée à une base de données, qui comprendrait les caractéristiques physiques (au sens large) du milieu, tes résultats des modèles utilisés, y compris les analyses de sensibilité, c'est-à-dire la finesse avec laquelle les paramètres ont été évalués sur ces milieux. La mise en œuvre de cette base ne sera certainement pas aisée, elle augmenterait fortement la crédibilité des études prédictives.

\section{Bibliographie}

Bourrain X. (1988). - Modélisation de l'autoépuration de l'Arc en aval d'Aix-en-Provence. Première partie. Recueil des données de terrain et interprétation. Rapport d'étude. Agence Rhône Méditerranée Corse. 55 pages.

Fisher H.B., LIST E.H., KoH R.Y.C., IMBERGER J. and Brooks N.H. (1979), - Mixing in inland and coastal waters. Academic Press, New York.

Jobson H.E. and Schoellhamer D.H. (1987). - Users manual for a branched lagrangian transport model. U.S. Geological Survey, Water-Resources Investigation Report 87-4163.

JoBson H.E. (1987). - Lagrangien model of Nitrogen kinetics in the Chattahoochee river. J. of Environmental Engineering, ASCE, 113, 223-242.

MCBRIDE G. and RUTHERFORD J.C. (1984). - Accurate modeling of river pollutant transport. J. of Environmental Engineering, ASCE, 110, 808-827.

O'KANE J.P.J. (1974). - A kinematic reference frame for estuaries of one dimension. "Mathematicals models in hydrology ", Symposium IAHR, Varsovie, 1971, 797807.

SChoellhamer D.H. (1988). - Lagrangian transport modelling with QUAL-II kinetics. J. of Environmental Engineering, ASCE, 114, 368-381.

SiMON L. (1989). - Evaluation de schémas numériques pour la modélisation de la qualité des eaux. "Les modèles mathématiques pour la gestion de la qualité des eaux superficielles ", Session S.H.F., novembre 1989, Paris. 